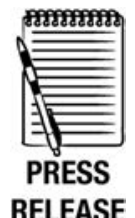

- An additional appendix is published online only. To view this file please visit the journa online (http://dx.doi.org/ 10.1136/tobaccocontrol-2011050147).

Department of Health Management and Policy, School of Public Health, University of Michigan, Ann Arbor, Michigan, USA

\section{Correspondence to}

Dr David Méndez, Department of Health Management and Policy, School of Public Health, University of Michigan, 1415 Washington Heights, Ann Arbor MI 48109-2029, USA dmendez@umich.edu

Received 15 July 2011 Accepted 15 March 2012

Published Online First 25 April 2012

\title{
The potential impact of smoking control policies on future global smoking trends
}

\author{
David Méndez, Omar Alshanqeety, Kenneth E Warner
}

\begin{abstract}
Background The authors develop projections for global smoking prevalence for the years 2020 and 2030 with and without the implementation, starting in 2010, of the WHO's recommended multipronged approach to tobacco control known as the MPOWER policy package.
\end{abstract}

Methods Using data from the WHO's Global InfoBase Database and the WHO's Global Adult Tobacco Survey, the authors construct adult cigarette smoking prevalence time series for 60 countries that account for $90 \%$ of the world's smokers and $85 \%$ of the world's population. The authors then use a stock/flow model to project those countries' smoking prevalence for the years 2020 and 2030, with and without the implementation of MPOWER. The authors aggregate the results and report regional and global figures.

Results The authors estimate global adult cigarette smoking prevalence in 2010 to be $23.7 \%$. If no additional policies are set in place and the initiation and cessation rates existing in 2010 persist, the authors estimate that global prevalence will be $22.7 \%$ by 2020 and $22.0 \%$ by 2030 (872 million smokers). If MPOWER had been implemented globally starting in 2010 with a $100 \%$ price increase for cigarettes, the authors estimate that global cigarette smoking prevalence would be $15.4 \%$ in 2020 and $13.2 \%$ in 2030 (523 million smokers).

Conclusions The estimates indicate the magnitude and trajectory of the global tobacco pandemic and of the impact the authors could expect if evidence-based tobacco control policies were applied immediately and universally throughout the world. As half of lifetime smokers die of tobacco-related diseases, if MPOWER were applied globally, within a few decades, many millions of premature tobacco-related deaths would be avoided.

Tobacco use, and in particular cigarette smoking, is the leading preventable cause of mortality around the world, responsible for a death toll in excess of 5 million people per year (more than HIV/AIDS, tuberculosis and malaria combined). The international community is increasingly aware of the impact of the tobacco epidemic on the health of the world population and is making serious efforts to control this global threat. In 2003, the World Health Assembly, the annual meeting of the member countries of the WHO, adopted the Framework Convention on Tobacco Control (FCTC), a treaty now ratified by 174 countries that have agreed to take steps to reduce both the supply of and demand for tobacco products. To help those countries fulfil their commitment to the FCTC, in 2008, the WHO disseminated recommendations for a multipronged approach to tobacco control consisting of six basic strategies: monitor tobacco use; protect people from tobacco smoke; offer help to quit tobacco use; warn about the dangers of tobacco; enforce bans on tobacco advertising, promotion and sponsorship; and raise taxes on tobacco. This comprehensive set of tobacco control policies is known as MPOWER. ${ }^{1}$ The package is intended to assist in the country-level implementation of effective measures to reduce the demand for tobacco, contained in the WHO FCTC.

Several global surveys have been implemented to monitor the course and patterns of tobacco use throughout the world and a number of studies report estimates of tobacco smoking prevalence in specific countries and world regions. ${ }^{1-6}$ While studies focus on country- and region-specific projections, ${ }^{7-9}$ the literature is very scant when it comes to predicting future global trends. ${ }^{10}$ This is especially true for projections incorporating the effects of implementing different tobacco control policies at the global level. In particular, while there is little disagreement that implementing the MPOWER policy package will reduce smoking rates worldwide, the magnitude of that potential reduction has not been previously estimated.

The aims of this study are to develop projections for global adult cigarette smoking prevalence for the years 2020 and 2030 with and without the implementation of the MPOWER package, based on available country-specific prevalence data and best estimates of the effectiveness of the tobacco control measures in the MPOWER policy set. To conduct our analysis, we focus on 60 countries comprising $85 \%$ of the world's population and $90 \%$ of global smoking prevalence. We first project what overall global prevalence in 2020 and 2030 would be if the trends observed in 2010 persist and no additional tobacco control policies are put in place. Then, we repeat the analysis assuming that, starting in 2010 all countries in the study apply the MPOWER policies, again developing global smoking projections for the years 2020 and 2030. We conduct extensive sensitivity analysis and compute most likely worstand best-case scenarios for our smoking prevalence projections. We include an online appendix with detailed information on the data sources we use and the data cleaning process we apply to each country included in the study. We also discuss in the online appendix, the methods we used to estimate the combined effect of individual policies.

\section{METHODS}

The analysis was divided into two parts: a) modelling of baseline prevalence and b) development of prevalence estimates for 2020 and 2030 if MPOWER policies were implemented in 2010. 
To develop the baseline prevalence estimates for 2020 and 2030, we first assembled prevalence time series for selected countries to compute the current trend of smoking rates. To construct the time series, we employed the tobacco use component of the WHO's Global InfoBase Database, a comprehensive compilation of historical tobacco use prevalence data in 191 countries. The countries are grouped into six main regions, corresponding to the official WHO regional divisions: AFRO (Africa), AMRO (the Americas), EMRO (the Eastern Mediterranean), EURO (Europe), SEARO (South-East Asia) and WPRO (the Western Pacific). The database includes prevalence rates obtained from published and non-published sources of governmental as well as non-governmental surveys. The database provides detailed information on each survey, including the definition of tobacco use (eg, current smokers vs. daily or eversmokers), tobacco type, age groups, gender and geographical representation (national vs sub-national). In each region, we chose to focus on the 10 countries with the largest number of current cigarette smokers. This group of 60 target countries accounts for around $90 \%$ of the world's smokers and $85 \%$ of the world's population. Table 1 shows the selected countries and each country's share of the global smoking population.

Table 1 Countries with largest percentage of world smokers (by region)

\begin{tabular}{|c|c|}
\hline & $\%$ Of global smokers \\
\hline \multicolumn{2}{|l|}{ AFRO } \\
\hline Nigeria & 0.6 \\
\hline South Africa & 0.6 \\
\hline Guinea & 0.3 \\
\hline Algeria & 0.3 \\
\hline Niger & 0.2 \\
\hline Mozambique & 0.2 \\
\hline Kenya & 0.2 \\
\hline United Republic of Tanzania & 0.2 \\
\hline Angola & 0.2 \\
\hline Uganda & 0.2 \\
\hline Subtotal & 3.2 \\
\hline \multicolumn{2}{|l|}{ EMRO } \\
\hline Pakistan & 1.6 \\
\hline Egypt & 1.3 \\
\hline Afghanistan & 0.8 \\
\hline Iran (Islamic Republic of Iran) & 0.6 \\
\hline Sudan & 0.6 \\
\hline Iraq & 0.3 \\
\hline Yemen & 0.3 \\
\hline Syrian Arab Republic & 0.3 \\
\hline Saudi Arabia & 0.3 \\
\hline Morocco & 0.3 \\
\hline Subtotal & 6.4 \\
\hline \multicolumn{2}{|l|}{ SEARO } \\
\hline India & 11.0 \\
\hline Indonesia & 4.8 \\
\hline Bangladesh & 1.7 \\
\hline Myanmar & 0.9 \\
\hline Thailand & 0.9 \\
\hline Democratic People's Republic of Korea & 0.4 \\
\hline Nepal & 0.3 \\
\hline Sri Lanka & 0.3 \\
\hline Timor-Leste & 0.0 \\
\hline Bhutan & 0.0 \\
\hline Subtotal & 20.3 \\
\hline
\end{tabular}

Continued
Table 1 Continued

\begin{tabular}{lc}
\hline & \% Of global smokers \\
\hline AMRO & \\
USA & 4.7 \\
Brazil & 1.9 \\
Mexico & 1.2 \\
Argentina & 0.7 \\
Peru & 0.5 \\
Colombia & 0.5 \\
Canada & 0.4 \\
Chile & 0.4 \\
Venezuela & 0.3 \\
Cuba & 0.2 \\
Subtotal & 10.9 \\
EURO & \\
Russian Federation & 4.0 \\
Germany & 1.7 \\
Turkey & 1.5 \\
Ukraine & 1.4 \\
France & 1.3 \\
UK & 1.1 \\
Spain & 1.1 \\
Italy & 0.9 \\
Poland & 0.9 \\
Romania & 0.5 \\
Subtotal & 14.3 \\
WPRO & \\
China & 28.0 \\
Japan & 2.6 \\
Philippines & 1.6 \\
Viet Nam & 1.3 \\
Republic of Korea & 1.0 \\
Malaysia & 0.4 \\
Australia & 0.3 \\
Cambodia & 0.2 \\
Lao People's Democratic Republic & 0.1 \\
Papua new guinea & \\
Subtotal & \\
Overall percentage prevalence & \\
\hline & \\
\hline
\end{tabular}

Using the raw (unadjusted) data from the InfoBase, we followed a multistage process to construct time trends for each of the target countries. First, we chose from among the surveys available in the InfoBase those that were conducted at the national level and reported overall prevalence rates for current cigarette smokers. Second, for those countries that had only a few surveys satisfying the criteria of the first stage, we looked for national surveys that provided prevalence rates for current smokers by gender and age categories. We then used the appropriate population estimates to derive a weighted average overall estimate for current smokers. Third, for countries where stages 1 and 2 result in insufficient data points to develop a trend, we made use of the available subnational data or data on daily smokers (daily smoker figures exclude people who are current smokers but do not smoke every day), making the necessary adjustments. This entailed the use of assumptions that varied by country and the available data. The data and process we used to construct the time series for each country are described in the online appendix.

Additionally, for selected countries, we supplemented the time trends with data from the WHO's Global Adult Tobacco Survey (GATS), ${ }^{11}$ a nationally representative household survey, launched in 2007 in 16 low- and middle-income countries that account for more than half of the world's smokers. These data 
are also shown in the online appendix. While in many countries, men and women exhibit dramatically different smoking patterns, due to the lack of sufficient data in several countries, we were not able to perform separate analyses for men and women.

To compute expected base case prevalence for the years 2020 and 2030, we adapted the method we have successfully used to model and forecast the natural trajectory of adult smoking prevalence in the USA to accommodate data from other countries. As in the US case, we modelled prevalence as a stock/flow process by keeping track of the accumulation over time of smokers who enter the system through smoking initiation and leave it due to smoking cessation or death. Our approach has been discussed extensively elsewhere. ${ }^{12-15}$ The following expression describes the basic process:

$$
\mathrm{dPrev}(\mathrm{t}) / \mathrm{dt}=\text { Init }-\operatorname{Prev}(\mathrm{t}) \times(\text { Cess }+ \text { Mortality })
$$

$$
\begin{aligned}
& \operatorname{Prev}(\mathrm{t})=\left(\operatorname{Prev}_{0}-(\text { Init } /(\text { Cess }+ \text { Mortality }))\right) \times \exp (-(\text { Cess } \\
& + \text { Mortality }) \times t)+(\text { Init } /(\text { Cess }+ \text { Mortality }))
\end{aligned}
$$

$\operatorname{Prev}(t)$ stands for adult smoking prevalence at time $t$ for the country under study; Init is the country-specific smoking initiation rate; Cess is the country-specific smoking cessation rate; Mortality is the country-specific mortality rate and Prev $_{0}$ is the estimated prevalence at the time we chose to initialise the country-specific time series.

To calibrate the model, we first obtained estimates for the adult initiation rate by averaging historical smoking prevalence at young ages (17-24) for each country with available data, thus assuming that little initiation occurs after age 24 . (We recognise that there is initiation after age 24 in some countries, which is a limitation of our study. In these countries, both prevalence and policy effects will be underestimated.) Then, for countries with a time series of two or more historical prevalence data points, we obtained estimates for the cessation rate and the initial prevalence $\left(\operatorname{Prev}_{0}\right)$, employing the model above and using non-linear least squares according to the following objective function:

$$
\begin{aligned}
& \operatorname{Min}_{\text {Cess,Prev0 }} \sum(\operatorname{Prev}(\text { Init, Cess, Prev }, \text { Mortality, } t) \\
& \text { - Observed Prevalence }(\mathrm{t}))^{2} .
\end{aligned}
$$

With the derived estimates, we used the model to project a smoking prevalence base case scenario for the years 2020 and 2030 for each country with available data, accounting for population growth. For countries with only one data point, we projected the same prevalence value to the years 2020 and 2030 as the base case but conducted a demanding sensitivity analysis around this assumption.

For each country in the study, we performed an extensive sensitivity analysis to develop worst- and best-case scenarios for the years 2020 and 2030. For countries with two or more observed prevalence data points, the sensitivity analysis consisted of conducting a Monte Carlo simulation to assess the variability of the 2020 and 2030 prevalence estimates according to the following procedure:

First, we fitted a triangular probability distribution to the data on initiation rates by selecting the minimum, maximum and mode statistics from the data as the distribution parameters. Then, we sampled from that distribution 10000 times, each time obtaining a new estimate of the cessation rate according to expression (3) and a projected prevalence for the years 2020 and 2030. Finally, we collected the distribution of projected prevalence values for 2020 and 2030 and reported the 2.5 and 97.5 percentiles as the best- and worst-case scenarios for smoking prevalence.

For the sensitivity analysis for countries with only one data point we assumed, as the worst-case scenario, that their smoking rates would follow the same upward trajectory as the USA prior to the 1960s. As such, we used as worst-case values for 2020 and 2030, the prevalence attained by the USA in 10 and 20 years, respectively, following the time when the USA reached the target country's most current prevalence estimate. As the highest prevalence attained by the USA was $<50 \%$ (in the late 1950 s), we did not compute a worst-case scenario for countries that are currently at $50 \%$ or higher smoking prevalence.

To estimate global smoking prevalence for the years 2020 and 2030 along with best- and worst-case scenarios, we conducted a Monte Carlo simulation sampling from the individual country distributions for the 2020 and 2030 prevalence obtained in the previous stage of the analysis. Each country's sample was then weighted by the country's adult population projection for 2020 and 2030 to obtain regional and overall world prevalence. ${ }^{16}$ The mean and the 2.5 and 97.5 percentiles were taken as the point estimate, best-case and worst-case estimate for the base case scenario of global prevalence in years 2020 and 2030.

To estimate the global impact of applying the smoking control policies described in the MPOWER package, we first obtained, from published sources, estimates of each policy's effectiveness on smoking initiation and cessation rates. ${ }^{17-19}$ We derived the estimate for the effectiveness of cessation support by taking the average of the effectiveness of different types of cessation support interventions reported by Wilson and colleagues. ${ }^{17}$ Assuming that just a fraction of current smokers will use cessation support, we then multiplied this average effectiveness by a cessation participation factor of $10 \%$, consistent with current literature, to obtain the 1.061 estimate we used in our calculations. The effectiveness values we used in our analysis are summarised in table 2 .

Except for the impact of taxes on smoking rates, values in table 2 show the improvement in initiation or cessation rates due to the application of individual MPOWER policies. For example, the table shows that a national mass media and package warning campaign would increase cessation rates by $23 \%$ and reduce initiation rates by $20 \%$. For the impact of taxation on smoking rates, the table reports the price elasticity of initiation rates and the price elasticity of smoking prevalence. For example, a tax increase that resulted in a $100 \%$ cigarette price increase would decrease prevalence by $20 \%$ within 1 or 2 years and produce a $70 \%$ reduction in initiation rates, both of which will be maintained as long as the real price reduction is sustained. We do not estimate reductions in daily smoking by continuing smokers.

We performed the policy impact analysis assuming that no MPOWER policies had been applied prior to 2010 and that in 2010, all MPOWER policies were implemented globally and simultaneously, with tax increases that would produce a $100 \%$ price increase for cigarettes. We also assume that this will be a real price increase, sustained throughout the time span of our analysis (ie, we assume that taxes will be adjusted for inflation annually to maintain the same proportional level above the price). We chose the $100 \%$ level as a plausible if ambitious target, 
Table 2 Effectiveness of MPOWER policies on initiation and cessation rates

\begin{tabular}{llll}
\hline Policy & Description & Effect on cessation (RR) & Effect on initiation (RR) \\
\hline P (protect) & Clean air laws & $1.11^{18}$ & $0.926^{17}$ \\
$O$ (offer help) & $\begin{array}{l}\text { Cessation support } \\
\text { W (warn) }\end{array}$ & $1.061^{17}$ & NA \\
Mass media and & $1.23^{17}$ & $0.8^{17}$ \\
E (enforce) & $\begin{array}{l}\text { package warnings } \\
\text { R (raise) }\end{array}$ & $1.03^{18}$ & $0.94^{18}$ \\
\hline
\end{tabular}

recognising that this would require an extremely large tax increase in nearly all countries.

We also considered different potential interactions among the individual policies that affect initiation and cessation rates when applied simultaneously. First, for a best-case scenario, we assumed that the policies acted independently on the population; that is, the fact that an individual is affected by one policy does not indicate that he/she is more or less likely to be affected by other policies. Discarding the possibility of negative correlation policy effects among individuals (ie, a person affected by one policy is less likely to be affected by other policies), the independence assumption provides the maximum combined effect of the policies on the 2020 and 2030 prevalence estimates, and thus, it constitutes our best-case scenario. We discuss this in greater detail in the online appendix.

For the worst-case scenario, we assumed maximum correlation among the effects of policies on individuals. This implies that the same subset of individuals is likely to be influenced by any policy. In this case, the maximum possible effectiveness from all policies combined is limited to that of the most effective policy.

Finally, for a more plausible intermediate case, we performed a Monte Carlo simulation sampling values for the combined policy effectiveness from a uniform distribution bounded by the best- and worst-case scenarios just described. On each iteration of the simulation, the sampled effectiveness modifies a pair of initiation and cessation rates resampled from the base case simulation run.

As in the base case scenario, the global prevalence for the years 2020 and 2030 is calculated by weighting the individual country estimates by their projected population size in 2020 and 2030, respectively. ${ }^{16}$

\section{RESULTS}

Table 3 shows our estimates of global and regional smoking prevalence for 2010, 2020 and 2030 under the base case setting (status quo). Table 4 shows the same information assuming that MPOWER policies are applied in 2010 and sustained thereafter. Each table present our estimates for best case, expected and worst case scenarios, as described in the Methods section.
We estimate global smoking prevalence in 2010 to be $23.7 \%$ (around 794 million smokers within the countries included in the study). If no additional policies are set in place and the initiation and cessation rates existing in 2010 persist, we estimate that global prevalence will be $22.7 \%$ by 2020 ( 838 million smokers, the larger number reflecting population growth) and $22.0 \%$ by 2030 ( 872 million smokers). Through our sensitivity analysis, we also estimate that global prevalence in 2030 could be as high as $22.7 \%$ or as low as $21.2 \%$.

Regional estimates of prevalence in 2010 range from 15.8\% in the AFRO region to $31.2 \%$ in the EURO region. Regional projections for 2020 and 2030 suggest that while the EURO, WPRO, AMRO and SEARO regions are trending down, smoking prevalence in the AFRO and EMRO regions will increase over the next 20 years under the status quo assumptions. The EURO region is still expected to have the highest smoking prevalence in 2030 (29.7\%), but the AFRO region is the one with the largest expected increase (from 15.8\% in 2010 to $21.9 \%$ in 2030).

Assuming that the MPOWER policies are applied and maintained globally starting in 2010 with a 100\% price increase for cigarettes, we estimate that global smoking prevalence will be around $15.4 \%$ in 2020 (569 million smokers) and 13.2\% in 2030 (523 million smokers), representing a $28 \%$ and $34 \%$ reduction in the number smokers from its 2010 value, respectively. The projected regional smoking prevalence for 2030 under the MPOWER policy package ranges from $8.9 \%$ (AMRO) to $17.0 \%$ (WPRO). The largest expected absolute decline corresponds to the EURO region (16.1 percentage points), while the AMRO region is expected to have the largest (57\%) proportional reduction in prevalence. The largest expected prevalence reduction attributable specifically to the MPOWER policies, both absolute (14.6 percentage points) and proportional (49\%), is projected to occur in the EURO region, moving from an expected prevalence in 2030 of $29.7 \%$ under the status quo to $15.1 \%$ with the application of MPOWER

\section{DISCUSSION}

Our results suggest that even if no additional tobacco control efforts are put in place between now and 2030, the world's overall adult smoking prevalence will decline slightly within

Table 3 Estimated smoking prevalence in 2020 and 2030 under base case scenario

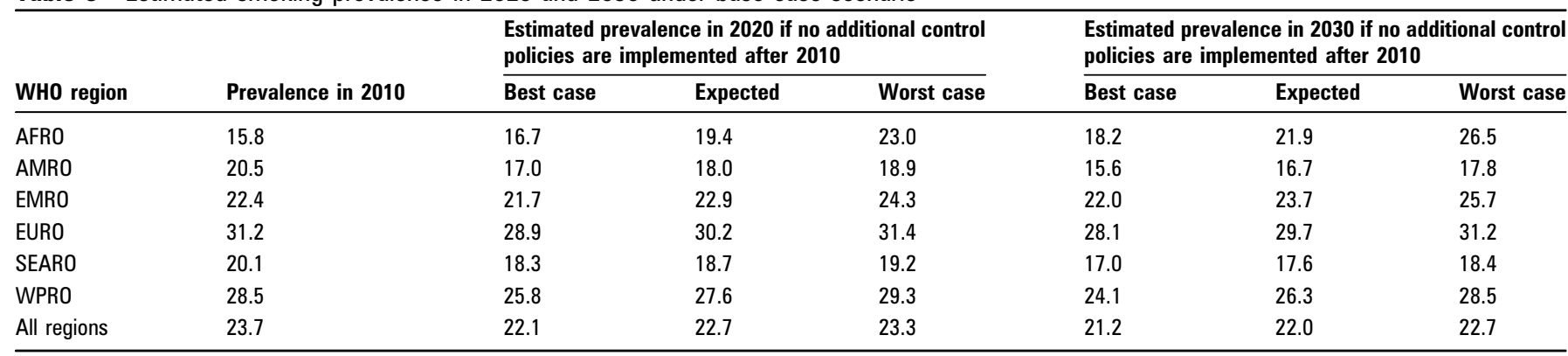


Table 4 Estimated prevalence in 2020 and 2030 under MPOWER policies with $100 \%$ price increase

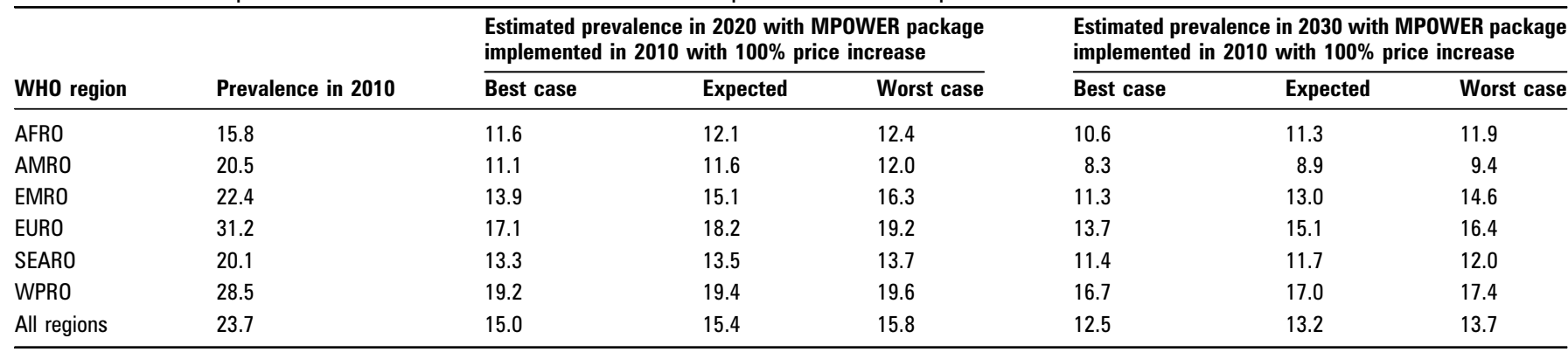

that period (from $23.7 \%$ to $22.0 \%$ ). This result is mixed news. On the one hand, it suggests that global smoking prevalence is not likely to rise over the next 20 years, but on the other hand, it also indicates that, due to expected population growth, the global number of smokers (at least those within the countries we studied, constituting approximately $90 \%$ of the world's smokers) will increase by $10 \%$, to a staggering 872 million smokers in 2030, from 794 million in 2010. Moreover, the stability of global prevalence can be deceiving, as different regions of the world are likely to exhibit substantial movement in smoking prevalence, albeit in different directions. As shown in table 2, while the Americas (AMRO), Europe (EURO), the Western Pacific (WPRO) and South-East Asian (SEARO) regions are trending down, the African (AFRO) and Eastern Mediterranean (EMRO) regions are trending up.

The potential impact of the MPOWER package is better news. Our results show that the application of the MPOWER package could set smoking prevalence on a downward trend in every region of the world. For the year 2020, our results show that universal application of the comprehensive set of policies, implemented fully in 2010, could decrease the global number of smokers by $28 \%$ over a 10 -year period. Over 20 years, the potential impact of MPOWER is much more dramatic, reducing the number of smokers by 271 million.

Our study is subject to several limitations. First, for several countries, we had very few historical prevalence data points with which to derive country-specific trends, as noted in the online appendix. Our estimates of future prevalence for these countries are subject to potentially large errors. Additionally, available surveys often employ very different methodologies and definitions of smoking even for the same country. It is important to promote a consistent and coherent data collection effort around the globe and particularly important in countries with rising smoking trends. Despite these data issues, as there is no obvious directional bias, and as we aggregated the country-specific results to perform regional analyses, we believe that our regional and global estimates of prevalence are reasonably robust. The results from our sensitivity analysis support this assertion. Second, our estimates of policy effectiveness are derived from studies in developed countries. The effect of the policies considered could differ in low- and middle-income countries. Third, we did not separate our analysis by gender. A substantial number of countries lacked the data to allow this distinction. In some countries, initiation patterns are very different for men and women; however, in most such countries, female smoking prevalence is very low, and thus, this modelling restriction does not significantly impact our results. Fourth, our model assumes that there is no initiation after age 24 . We recognise that this assumption is violated in some countries. In such cases, both prevalence and policy effects will be underestimated. Fifth, our analysis focuses strictly on cigarette smoking, ignoring other tobacco products. In countries such as India, other products represent a substantial amount of tobacco consumption. We chose to focus on the most important global tobacco-related source of disease-cigarette smoking-and the one that is by far the most common in the vast majority of countries. It is also the form of tobacco use for which prevalence data are most widely available and for which policy impacts are best understood.

It is important to emphasise that our assumption of doubling of price accounts for the majority of the impact of MPOWER policies (results not shown). This is particularly important to appreciate because in this study, we assume that the increase in real price will be maintained throughout the entire period of analysis, that is, prices would be adjusted annually to reflect inflation. To the extent that this did not occur, the effects of the one-time doubling of price would erode as inflation reduced the real value of the price. We also note that our sensitivity analysis focuses on the uncertainty stemming from the prevalence data. We did not perform sensitivity analysis around individual policy effectiveness, which we took as single estimates from the literature, reported in table 2 .

In our analysis, we assume that the MPOWER policies are applied globally and simultaneously in 2010, without a subsequent reaction from the tobacco industry to try to counteract the effect of those policies (eg, the industry's encouraging cigarette smuggling from low- to high-price countries). As such, our results should not be taken as forecasts, which are based on what are believed to be realistic assumptions of what will occur in the future. Rather, our estimates indicate the magnitude and trajectory of the global smoking pandemic and of the impact we could expect if evidence-based tobacco control policies were applied immediately and universally throughout the world. A final caveat, however: our estimates may exaggerate the potential decrease in smoking attributable to complete adoption of MPOWER because many countries implemented components of the MPOWER policy package prior to 2010. However, two factors mitigate the bias in our assumption: first, the largest policy effects are due to price increases, and such effects can always be attained regardless of prior price levels and second, in many countries, we have used data prior to the implementation of such policies to compute smoking trend. Additionally, our projections do not consider the possibility of a synergistic effect among policies acting simultaneously, which may lead to an underestimation of the MPOWER effects.

In an article published recently in The Lancet, ${ }^{20}$ the authors propose a target of $<5 \%$ for global prevalence in 2040. Our analysis suggests that under what we consider the highly unrealistic assumption of complete and instant adoption of MPOWER, with a $100 \%$ increase in cigarette price, the best possible scenario for global prevalence in 2030 will be $12.5 \%$. Reaching $5 \%$ in 2040 would be extremely difficult if we are constrained to our current policy arsenal. This is consistent with 


\section{What this paper adds}

This paper describes the global trajectory of adult smoking prevalence over the next 20 years and estimates the impact of applying globally, WHO recommended tobacco control policies.

the premise set forth by Warner and Méndez, ${ }^{21}$ who argue that without substantial innovation in tobacco control policy, further reductions in smoking in developed nations will come frustratingly slowly.

Nonetheless, the important message of our study is that the application of MPOWER globally would produce a substantial reduction in global cigarette smoking. If we assume that MPOWER strategies have similar effects on other tobacco product use, the reduction in global tobacco consumption could be much greater. As approximately half of lifetime smokers die of tobacco-related diseases, the implementation of MPOWER would prevent many millions of premature tobacco-related deaths. The Framework Convention on Tobacco Control can and should serve as the mechanism to move towards adoption of these policies. If implemented as required in the FCTC, the MPOWER strategies will contribute to significant declines in cigarette smoking over the next 20 years. However, the MPOWER strategies alone are not enough. We must fully implement all aspects of the FCTC and then continue to search for mechanisms to further drive down the use of tobacco.

Acknowledgements The authors would like to thank Simone Singh and Sejica Kim for their valuable contributions in constructing the individual countries' time series depicted in the online appendix.

Contributors Dr DM designed the study and contributed to the interpretation of the results and the writing of the manuscript. Dr OA carried out the analysis and contributed to the design of the study and the writing of the manuscript. Dr KEW contributed to the design of the study, the interpretation of the results and the writing of the manuscript.

Funding This study was supported by a grant from the International Union Against Tuberculosis and Lung Disease (IUATLD) made possible by support from Bloomberg Philanthropies.

Correction notice This article has been corrected since it was published Online First. The Funding statement has been updated.

Competing interests None.
Provenance and peer review Not commissioned; externally peer reviewed.

Data sharing statement All the data used to perform the analyses are available in an accompanying online appendix.

\section{REFERENCES}

1. The World Health Organization. MPOWER: A Policy Package To Reverse The Tobacco Epidemic. http://www.who.int/tobacco/mpower/mpower_english.pdf (accessed 5 Apr 2012).

2. Warren C, Lee J, Lea V, et al. Evolution of global tobacco surveillance system (GTSS) 1998-2008. Glob Health Promot 2009;2(Suppl):4-37.

3. Warren C, Jones N, Eriksen M, et al. Patterns of global tobacco use in young people and implications for future chronic disease burden in adults. Lancet 2006;367:749-53.

4. Pample F. Tobacco use in Sub-Saharan Africa: estimates from the demographic health surveys. Soc Sci Med 2008;66:1772-83.

5. Hammond KS. Global patterns of nicotine and tobacco consumption. In: Henningfield JE, London ED, Pogun S, eds. Nicotine Psychopharmacol. Berlin Heidelberg: Springler-Verlag, 2009:3-28.

6. Mackay J, Eriksen M, Shafey 0. The Tobacco Atlas. 2nd edn. Geneva: World Health Organization, 2006.

7. Levy DT, Cho SI, Kim YM, et al. SimSmoke model evaluation of the effect of tobacco control policies in Korea: the unknown success story. Am J Public Health 2010;100:1267-73

8. Levy DT, Ross H, Zaloshjna E. The Albania SimSmoke tobacco policy simulation model. Cent Eur J Public Health 2008;16:189-98.

9. Levy DT, Sarunya B, Ross H, et al. The role of tobacco control policies in reducing smoking and deaths in a middle income nation: results from the Thailand SimSmoke simulation model. Tob Control 2008;17:53-9.

10. Mackay J. The global tobacco epidemic: the next 25 years. Public Health Rep 1998;113:14-21

11. The World Health Organization. GATS (Global Adult Tobacco Survey). http:// www.who.int/tobacco/surveillance/gats/en/index.html (accessed 5 Apr 2012).

12. Mendez D, Warner KE, Courant PN. Has smoking cessation ceased? Expected trends in the prevalence of smoking in the United States. Am J Epidemiol 1998;148:249-28

13. Mendez D, Warner KE. Smoking prevalence in 2010: why the healthy people goal is unattainable. Am J Public Health 2000;90:401-3.

14. Mendez D, Warner KE. Adult cigarette smoking prevalence: declining as expected (not as desired). AJPH 2004;94:251-2.

15. Mendez D, Warner KE. Setting a challenging yet realistic smoking prevalence target for healthy people 2020: learning from the California experience. AJPH 2008:98:556-9.

16. U.S. Census Bureau. International Database (IDB). http://www.census.gov/ipc/ www/idb/informationGateway.php (accessed 16 Jan 2011).

17. Wilson LM, Tang EA, Chander G, et al. Impact of tobacco control interventions on smoking initiation, cessation and prevalence: a systematic review. J Environ Public Health [In press].

18. Levy DT, Gitchell JG, Chaloupka F. The effects of tobacco control policies on smoking rates: a tobacco control Scorecard. J Public Health Manag Pract 2004;10:338-51.

19. IARC. IARC Handbooks of Cancer Prevention: Tobacco Control. Effectiveness of Price And Tax Policies For Control Of Tobacco. Vol. 14. Lyon, France: International Agency for Research on Cancer. In press.

20. Beaglehole R, Bonita R, Horton $\mathrm{R}$, et al. Priority actions for the non-communicable disease crisis. Lancet 2011;377:1438-47.

21. Warner KE, Mendez D. Tobacco control policy in developed countries: yesterday, today, and tomorrow. Nicotine Tob Res 2010;9:876-87. 